BMJ Surgery, Interventions, \& Health Technologies

\section{Race and prostate imaging: implications for targeted biopsy and image-based prostate cancer interventions}

To cite: Gross MD, Al Hussein Al Awamlh B, Shoag JE, et al. Race and prostate imaging: implications for targeted biopsy and image-based prostate cancer interventions. BMJ Surg Interv Health Technologies 2019;1:e000010. doi:10.1136/ bmjsit-2019-000010

Received 25 March 2019 Accepted 06 June 2019

Check for updates

(C) Author(s) (or their employer(s)) 2019. Re-use permitted under CC BY-NC. No commercial re-use. See rights and permissions. Published by BMJ.

${ }^{1}$ Urology, Weill Cornell Medical College, New York, New York, USA

${ }^{2}$ Healthcare Policy and Research, Weill Cornell Medical College, New York, New York, USA

${ }^{3}$ Radiology, Weill Cornell Medical College, New York, New York, USA

${ }^{4}$ Pathology, Weill Cornell Medical College, New York, New York, USA

${ }^{5}$ Preventative Medicine, Keck School of Medicine of the University of Southern California, Los Angeles, California, USA

${ }^{6}$ Bureau of Cancer Epidemiology, New York State Department of Health, Albany, New York, USA

Correspondence to

Jim C Hu;

Jch9011@med.cornell.edu

\section{ABSTRACT}

Purpose For men with an elevated prostate-specific antigen (PSA), there is a strong evidence for prostate MRI to assess the risk of clinically significant prostate cancer (CSPC) and guide targeted-biopsy interventions. Prostate MRI is assessed using the Prostate Imaging-Reporting and Data System (PI-RADS), which is scored from 1 to 5. Equivocal or suspicious findings (PI-RADS 3-5) are recommended for subsequent targeted biopsy, for which the risk of infection and sepsis is increasing. However, PI-RADS was developed primarily in men of European descent. We sought to elucidate PI-RADS and MRItargeted biopsy outcomes in Asian men, a rapidly growing population in the USA, Europe, Australia and internationally. Materials and methods A prospective cohort of 544 men with elevated PSA without a diagnosis of prostate cancer who underwent MRI-targeted biopsy at our institution from January 2012 to December 2018 was analyzed. We categorized the cohort by self-designated race then used a validated algorithm which uses surname lists to identify a total of $78(14 \%)$ Asian-Americans. The primary outcome was the likelihood of diagnosing CSPC (Gleason grade group >1) in Asian-Americans versus non-AsianAmericans. Multivariable logistic regression was used to determine the association of demographic and other characteristics with CSPC.

Results Overall, MRI-targeted biopsy identified CSPC in $17 \%$ of Asian-American men versus $39 \%$ of non-AsianAmerican men $(p<0.001)$. Notably for PI-RADS 3, only $6 \%$ of Asian-Americans versus $15 \%$ of others were diagnosed with CSPC. In adjusted analyses, Asian-American men were less likely to be diagnosed on MRI-targeted biopsy with CSPC (OR $0.30,95 \% \mathrm{Cl} 0.14$ to $0.65, \mathrm{p}=0.002)$ and indolent prostate cancer (OR $0.37,95 \% \mathrm{Cl} 0.15$ to 0.91 , $p=0.030$ ) than other races. Regardless of race those who were biopsy naïve were more likely (OR $2.25,95 \% \mathrm{Cl} 1.45$ to $3.49, p<0.001$ ) to be diagnosed with CSPC.

Conclusion We found that PI-RADS underperforms in Asian-American men. For instance, only 2 of 35 (6\%) Asian-American men with PI-RADS 3 were diagnosed with CSPC on MRI targeted biopsy. This has significant implications for overuse of diagnostic and image-guided interventional approaches in Asian-Americans, given the increasing risk of infectious complications from biopsy. Additional validation studies are needed to confirm our findings.

\section{Key messages}

What is already known on the subject?

- MRI aids in assessing the risk for clinically significant prostate cancer and for targeting suspicious areas seen on imaging within the prostate. The utility of prostate MRI as a biomarker for elevated PSA in Asians has not been assessed.

What are the new findings?

- This study finds that Asian men have a significantly lower risk of finding significant cancer at every risk score for prostate MRI.

\section{How might these results affect future} research or surgical practice?

- This highlights the need to assess the generalizability of biomarkers beyond the original race in which they were validated, in keeping with the IDEAL framework. We may begin to consider avoidingprostate biopsy in some Asian men if other risk factors are favorable, and our findings have significant implications for prostate MRI and targeted ablation.

\section{INTRODUCTION}

There is strong evidence supporting the use of prostate MRI for men with elevated prostate-specific antigen (PSA) to diagnose and guide treatment of prostate cancer. ${ }^{1}$ Prostate MRI is assessed using the Prostate Imaging-Reporting and Data System (PI-RADS) which grades the likelihood of clinically significant cancer on a 1-5 scale. Men with prostate MRI assigned PI-RADS scores 3-5 are recommended for biopsy. This scoring system was developed in men of European descent. ${ }^{1}$ In addition to risk stratification, prostate MRI aids in directing ultrasound-guided transrectal biopsy through the use of MRI-ultrasound fusion software. ${ }^{2}$ Recent prospective randomized trials demonstrate better detection of clinically significant prostate cancer with MRI-targeted biopsy as compared with standard transrectal ultrasound-guided biopsy. ${ }^{2-4}$ Neither the PRECISION (Prostate Evaluation 
for Clinically Important Disease: Sampling Using Image Guidance or Not?) nor the MRI-FIRST randomized trials report patient race, but given the predominantly European study sites, non-white patient representation is likely very low. ${ }^{45}$ This may have significant implications as MRI and targeted biopsy are disseminated worldwide.

Following the Idea, Development, Exploration, Assessment, Long-term monitoring (IDEAL) framework, which seeks to improve the quality of research in surgery and devices through staged development and analysis of procedures, we consider targeted biopsy evidence development in the long-term monitoring surveillance phase. ${ }^{6}$ Real-world evidence, audits and risk adjustments are required to further investigate current practices, especially in understudied populations. In this context the US Preventive Services Task Force and other professional societies have emphasized a need for research to bridge the significant evidence gap in disparities regarding prostate cancer presentation and outcomes in under-represented populations. ${ }^{7}$ The relationship between MRI findings and biopsy outcomes has not been thoroughly explored in Asian-Americans. The Asian-American population is the fastest growing racial group in the USA and many Western countries. The Asian-American population is projected to double from 18.3 to 36.8 million by 2060, at which time Asian-Americans will comprise the largest immigrant group. ${ }^{8}$ Racial disparities in prostate cancer incidence have been reported, with Asian-Americans having a prostate cancer incidence rate of 67.8 per 100000 men as compared with 123.0 in non-Hispanic whites. $^{9}$

With an increasing rate of infection following prostate biopsy, the diagnostic yield of prostate biopsy must be balanced against procedural harm. ${ }^{10-12}$ Moreover, prostate MRI and targeted biopsy are integral to current diagnostic and therapeutic paradigms that hinge on accurate risk stratification for treatment selection and the emerging use of partial gland ablation for prostate cancer. In this study, we examine MRI-targeted biopsy outcomes for an elevated PSA in among Asian-American versus non-Asian-American men.

\section{MATERIALS AND METHODS}

We analyzed our prospective database of men with elevated PSA without a diagnosis of prostate cancer who underwent multiparametric MRI and subsequent targeted biopsy at Weill Cornell Medicine from 2012 to 2018. The MRI and biopsy protocol has been previously described. ${ }^{13}$ Prostate MRI was evaluated using PI-RADS version 2, and men were categorized according to their highest rated region of interest. ${ }^{14}$ MRI studies which occurred prior to the release of PI-RADS version 2 in 2015 were retrospectively recategorized by an experienced uroradiologist (DJM). A flow diagram of the included patients is presented in figure 1.

All biopsies were performed using the Artemis MRI-ultrasound fusion-targeted biopsy platform (Eigen, Grass
Patients undergoing MRI-targeted biopsy during 2012-2018 $(n=718)$

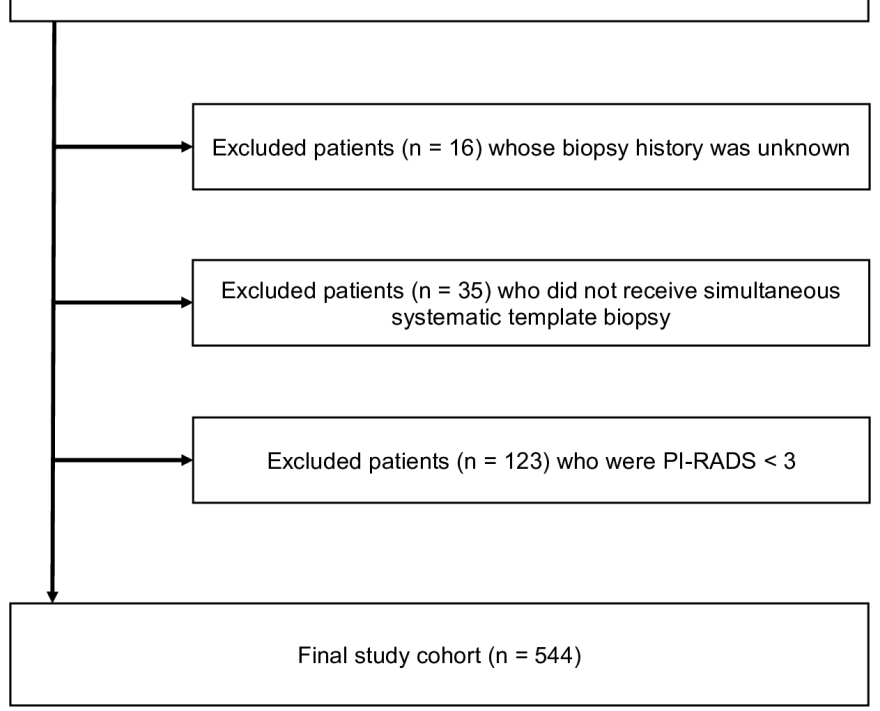

Figure 1 Patient selection and exclusion criteria.

Valley, CA, USA). We excluded all subjects with PI-RADS classification less than 3. Regardless of race, these men generally do not receive biopsy at our institution. Biopsies included at least one targeted core per lesion, as well as systematic cores. All biopsy cores were reviewed for determination of the highest pathological grade by an experienced genitourinary pathologist. Clinically significant prostate cancer was defined as Gleason grade group $>1$.

There was a significant number of patients who declined to self-report their race $(n=108)$. Therefore, we relied on both self-reporting as well as surname analysis to identify Asian-Americans in the cohort. Fifty-three men self-reported as Asian-American, and given and surname analysis using a method by Lauderdale and Kestenbaum identified 25 additional Asian-Americans. ${ }^{15}$ This method used lists of names provided by the authors that are generated from Social Security Administration records, which include country of birth, and are validated against census reports with positive predictive value between $82 \%$ and $98 \%$. Of note, $94 \%(50 / 53)$ who self-identified in our cohort were validated using the surname analysis.

The primary study outcome was detection of clinically significant prostate cancer. The secondary outcomes were clinically significant prostate cancer detection among PI-RADS 3-5 categories, the detection of indolent prostate cancer (Gleason grade group 1), and cancer detection by targeted versus systematic biopsy cores. Systematic cores which overlapped with a region of interest during fusion biopsy were retrospectively reclassified as targeted cores in a manner previously described. ${ }^{16}$

Patient-level variables were analyzed using MannWhitney U tests and $\chi^{2}$ tests. Descriptive variables collected include race, age, body mass index, PSA, prostate volume by MRI, PSA density, PI-RADS category, and prior negative versus first time biopsy. When the event rate for detection of cancer was less than or equal to 5, the Fisher's exact 
Table 1 Study characteristics

\begin{tabular}{lllc}
\hline & Asian-American & Other & P value \\
\hline $\begin{array}{l}\text { Patients, } \mathrm{n} \\
\text { Median }(\mathrm{IQR})\end{array}$ & 78 & 466 & \\
\hline Age (year) & & & \\
Body mass index $\left(\mathrm{kg} / \mathrm{m}^{2}\right)$ & $67.1(63-71.2)$ & $66.7(60.1-71.8)$ & 0.653 \\
\hline PSA $(\mathrm{ng} / \mathrm{mL})$ & $24.9(22.8-27)$ & $27.0(25-29.7)$ & $<0.001$ \\
\hline Prostate volume $\left(\mathrm{cm}^{3}\right)$ & $6.9(4.8-10.7)$ & $6.3(4.6-9.5)$ & 0.368 \\
\hline PSA density $\left(\mathrm{ng} / \mathrm{mL}^{\mathrm{c}} \mathrm{cm}^{3}\right)$ & $45.2(33-73)$ & $51.2(36.6-73.1)$ & 0.407 \\
\hline Lesion diameter $(\mathrm{cm})$ & $0.16(0.07-0.24)$ & $0.12(0.08-0.19)$ & 0.027 \\
$\mathrm{n}(\%)$ & $1.1(0.6-1.5)$ & $1.1(0.7-1.5)$ & 0.276 \\
\hline Biopsy naïve & & & 0.002 \\
\hline PI-RADS 3 & $30(38)$ & $269(58)$ & 0.146 \\
\hline PI-RADS 4 & $35(45)$ & $162(35)$ & \\
\hline PI-RADS 5 & $32(41)$ & $203(44)$ & \\
\hline
\end{tabular}

PI-RADS, Prostate Imaging-Reporting and Data System; PSA, prostate-specific antigen.

test was used in lieu of the $\chi^{2}$ test. Comparison of systematic and targeted biopsy yield was assessed via McNemar's test for equivalence. Multivariable logistic regression was performed to assess the association of Asian-American race on detection of clinically significant prostate cancer and indolent prostate cancer. Age and body mass index were treated as continuous variables. PI-RADS category, PSA density, and biopsy history were treated as categorical variables with respect to appropriate referents. SPSS V.25 (IBM) was used for statistical analyses.

\section{RESULTS}

Demographic and clinical characteristics are presented in table 1 . Of the 544 men included in this study, $78(14 \%)$ were Asian-American, 283 (52\%) Caucasian, 45 (8\%) African-American, 31 (6\%) Hispanic, and 107 (20\%) were of unknown/other race. Asian-American men had lower body mass index compared with non-Asian-Americans (median 24.9 vs $27.0 \mathrm{~kg} / \mathrm{m}^{2}, \mathrm{p}<0.001$ ). More Asian-Americans had undergone a prior negative biopsy ( $62 \%$ vs $42 \%$, $\mathrm{p}=0.002)$. PSA density was greater in the Asian-American group ( 0.16 vs $0.12 \mathrm{ng} / \mathrm{mL} / \mathrm{cm}^{3}, \mathrm{p}=0.027$ ). Age, PSA and prostate volume did not vary by race. There was no significant difference in the distribution of PI-RADS categories between the two groups.

Overall, MRI-targeted biopsy detected clinically significant prostate cancer in $17 \%$ of Asian-Americans versus $39 \%$ of non-Asian-Americans $(\mathrm{p}<0.001)$. Table 2 shows the biopsy results across different PI-RADS classifications. Asian-Americans with a PI-RADS 4 lesion were significantly less likely to be diagnosed with clinically significant prostate cancer compared with non-Asian-Americans $(16 \%$ vs $44 \%, \mathrm{p}=0.003$ ). For indolent cancer, diagnoses occurred in $8 \%$ of Asian-Americans vs $21 \%$ of non-Asian-Americans $(\mathrm{p}=0.006)$. This difference was also significant for PI-RADS 3 (6\% vs 22\%, p=0.031). Figure 2 depicts the proportion of biopsy-proven cancer among Asian-Americans and non-Asian-Americans.

Table 3 shows the targeted and systematic biopsyyield, after accounting for systematic cores overlapping with targeted biopsy of the region(s) of interest. In both Asian-Americans and non-Asian-Americans, targeted biopsy was superior for the detection of clinically significant prostate cancer $(15 \%$ vs $6 \%, \mathrm{p}=0.0455$ and $33 \%$ vs $10 \%, \mathrm{p}<0.001$ respectively). Only one Asian-American (1\%) was diagnosed with clinically significant prostate cancer on systematic biopsy where targeted biopsy identified indolent prostate cancer. In the non-Asian-American cohort, 7 (1.5\%) men were diagnosed with clinically significant prostate cancer on systematic biopsy while targeted biopsy showed no cancer $(n=2)$ or indolent cancer $(n=5)$.

Table 2 Cancer detection by PI-RADS score and overall

\begin{tabular}{|c|c|c|c|}
\hline & $\begin{array}{l}\text { Asian- } \\
\text { American, } \\
\mathrm{n}(\%)\end{array}$ & $\begin{array}{l}\text { Other, n } \\
\text { (\%) }\end{array}$ & $P$ value \\
\hline \multicolumn{4}{|c|}{ Clinically significant prostate cancer } \\
\hline PI-RADS 3 & $2(6)$ & $25(15)$ & 0.177 \\
\hline PI-RADS 4 & $5(16)$ & $89(44)$ & 0.003 \\
\hline PI-RADS 5 & $6(55)$ & $68(67)$ & 0.505 \\
\hline Overall & $13(17)$ & 182 (39) & $<0.001$ \\
\hline \multicolumn{4}{|c|}{ Indolent prostate cancer } \\
\hline PI-RADS 3 & $2(6)$ & $36(22)$ & 0.031 \\
\hline PI-RADS 4 & $4(13)$ & $46(23)$ & 0.248 \\
\hline PI-RADS 5 & $0(0)$ & $16(16)$ & 0.360 \\
\hline Overall & $6(8)$ & $98(21)$ & 0.006 \\
\hline
\end{tabular}

PI-RADS, Prostate Imaging-Reporting and Data System. 


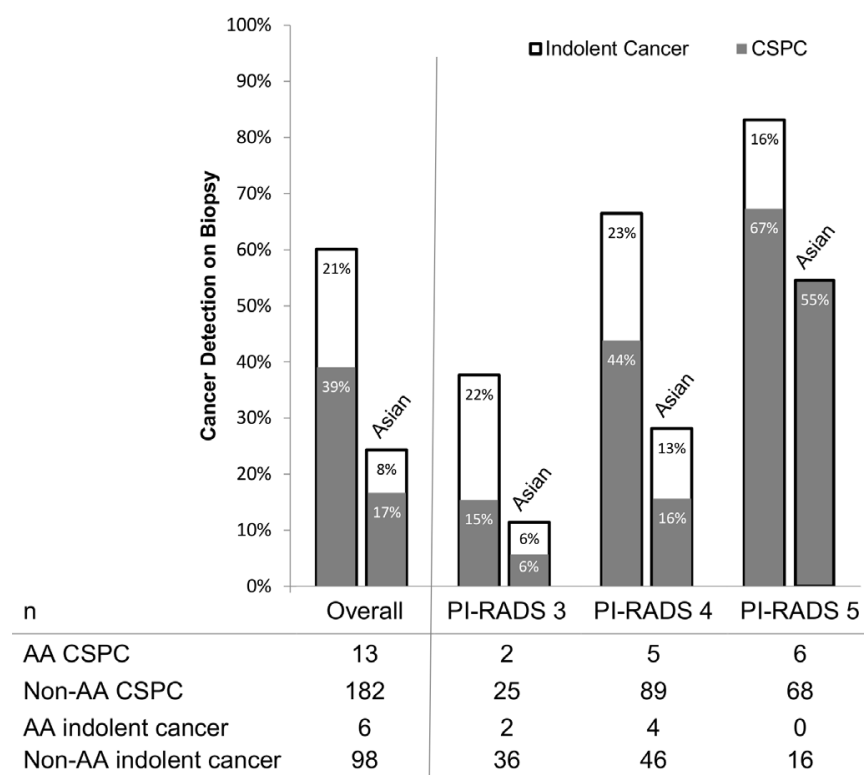

Figure 2 Prostate Imaging-Reporting and Data System (PIRADS) and detection of prostate cancer by race. AA, AsianAmerican; CSPC, clinically significant prostate cancer.

In multivariable logistic regression, Asian-Americans were less likely to be diagnosed with clinically significant prostate cancer on MRI-targeted biopsy compared with non-Asian-Americans (OR $0.30,95 \%$ CI 0.14 to $0.65, \mathrm{p}=0.002)$. In addition, age (OR 1.07, 95\% CI 1.04 to $1.11, \mathrm{p}<0.001$ ), PSA density (OR 4.02, 95\% CI 2.57 to $6.29, \mathrm{p}<0.001)$, and biopsy naïve status versus prior negative biopsy (OR 2.25, 95\% CI 1.45 to 3.49 , p<0.001) were associated with detection of clinically significant prostate cancer. The PI-RADS categories 4 (OR 3.82, 95\% CI 2.27 to $6.45, \mathrm{p}<0.001$ ) and 5 (OR 7.69, 95\% CI 4.14 to 14.30 , $\mathrm{p}<0.001)$ had strong associations with clinically significant prostate cancer relative to PI-RADS 3 (table 4). Asian-Americans were also less likely to be diagnosed

Table 3 Comparison of biopsy result for targeted and systematic biopsy

\begin{tabular}{lcccc}
\hline \multirow{2}{*}{$\begin{array}{l}\text { Systematic } \\
\text { biopsy }\end{array}$} & $\begin{array}{l}\text { Targeted biopsy } \\
\text { No PCa }\end{array}$ & $\begin{array}{l}\text { Indolent } \\
\text { PCa } \mathbf{n}(\%)\end{array}$ & $\begin{array}{l}\text { CSPC } \mathbf{n} \\
(\%)\end{array}$ & Total \\
\hline Asian-American & & & & \\
\hline No PCa & $62(79)$ & $3(4)$ & $8(10)$ & 73 \\
Indolent PCa & $0(0)$ & $0(0)$ & $0(0)$ & 0 \\
\hline CSPC & $0(0)$ & $1(1)$ & $4(5)$ & 5 \\
\hline Total & 62 & 4 & 12 & 78 \\
Other & & & & \\
\hline No PCa & $239(51)$ & $45(10)$ & $101(22)$ & 385 \\
Indolent PCa & $12(3)$ & $13(3)$ & $8(2)$ & 33 \\
\hline CSPC & $2(0)$ & $5(1)$ & $41(9)$ & 48 \\
\hline Total & 253 & 63 & 150 & 466 \\
\hline
\end{tabular}

CSPC, clinically significant prostate cancer; $\mathrm{PCa}$, prostate cancer. with indolent prostate cancer (OR $0.37,95 \%$ CI 0.15 to $0.91, \mathrm{p}=0.030$ ). Older age was associated with fewer diagnoses of indolent cancer (OR 0.97, $95 \%$ CI 0.945 to 0.999 , $\mathrm{p}=0.045$ ).

\section{DISCUSSION}

To our knowledge, this is the first study to examine MRI-targeted biopsy performance in Asian-Americans. We demonstrate that PI-RADS underperforms in Asian-Americans versus non-Asian-Americans. In this racial group, clinically significant prostate cancer was more than two-thirds less likely to be diagnosed as compared with men of other races. This has significant implications for the potential overuse of biopsy and its harms at the population level.

Epidemiologic studies have observed a lower incidence of prostate cancer and prostate cancer-specific mortality in men of Asian-American versus non-Asian-American descent. ${ }^{17} 18$ These differences have been attributed to socioeconomic status and access to care. However, a prostate epidemiology study in an equal-access population demonstrated lower clinical stage and improved pathological outcomes for Asian-Americans as compared with other races. ${ }^{19}$ One may circumvent screening bias entirely by considering cases of prostate cancer found incidentally on autopsy. In this setting, Japanese immigrants to Hawaii demonstrated a significantly lower incidence of latent prostate cancer $(25.6 \%)$ as compared with African-Americans $(36.9 \%)$ and Caucasians $(34.6 \%) .{ }^{20}$ Both dietary and genetic factors have also been proposed to explain these trends. $^{21-23}$

Only $6 \%$ of Asian-Americans with a PI-RADS 3 region of interest were diagnosed with clinically significant prostate cancer. This is in contrast to the $15 \%$ rate observed in non-Asian-Americans which is consistent with published rates of $12 \%-21 \%$ for PI-RADS $3 .{ }^{34}$ Similarly, Asian-Americans also had half the incidence of indolent cancers compared with others. Our findings emphasize the importance of examining the generalizability of new medical technologies and diagnostic strategies such as PI-RADS and MRI-targeted biopsy in different populations.

Our finding that older age is associated with an increased likelihood of clinically significant prostate cancer is consistent with data from the Cancer of the Prostate Strategic Urologic Research Endeavor (CaPSURE) demonstrating an association between age and high-grade disease. ${ }^{24} \mathrm{~A}$ similar association has also been found in other population-based studies. ${ }^{25} 26$

As expected, men with a prior negative biopsy had a lower likelihood of finding clinically significant prostate cancer. $^{27}$ Of note, only 6/48 (13\%) of Asian-American men with a prior negative biopsy were diagnosed with clinically significant prostate cancer in contrast to 54/197 $(27 \%)$ of non-Asian-Americans $(p=0.031)$. Only one Asian-American man with a prior negative biopsy was diagnosed with clinically significant disease from a PI-RADS 3 region of interest, finding $<5 \%$ Gleason pattern 4 . 
Table 4 Logistic regression for factors associated with MRI-targeted biopsy diagnosis of indolent and clinically significant prostate cancer

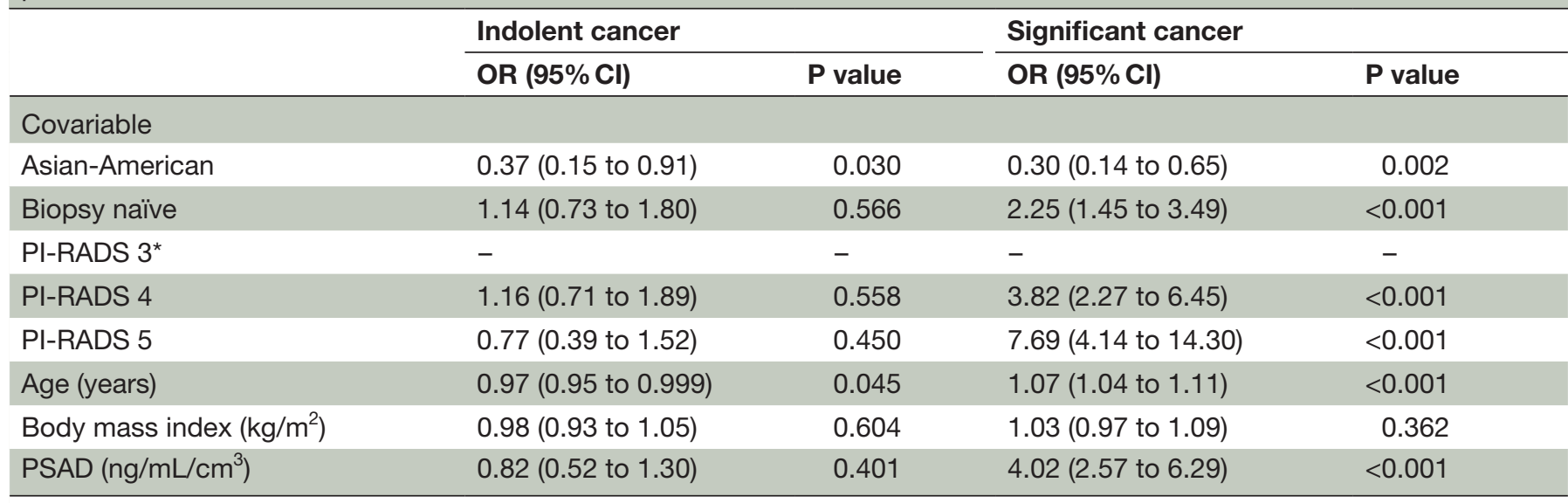

Non-AA is the referent for AA. Prior negative biopsy is the referent for biopsy naive.

${ }^{*} \mathrm{PI}$-RADS 3 as referent.

†PSAD $<0.15$ as referent.

AA, Asian-American; PI-RADS, Prostate Imaging-Reporting and Data System; PSAD, prostate-specific antigen density.

Our findings exemplify the significance of the 'long-term monitoring' IDEAL stage 4 for a commonly performed invasive intervention that has diagnostic intent for the most common solid organ tumor in Western countries. ${ }^{6}$ Despite strong evidence demonstrating better outcomes of a new technological innovation, real-world evidence must be gathered to assess its effectiveness beyond the population in which it was developed and validated. As use of prostate MRI has grown in recent years, estimates show its adoption as a reflex test for elevated PSA would contribute billions of healthcare dollars annually. ${ }^{28}$ Our finding of low performance of PI-RADS 3 for Asian-Americans in an experienced center has significant implications for prostate cancer screening and biopsy overuse in men of Asian descent. For instance, a higher PI-RADS threshold for MRI-targeted biopsy in Asian-Americans may be more cost-effective and spare many the morbidity of biopsy, particularly with the significant increase in the infectious complications of prostate biopsy, ${ }^{10-12}$ MRI-targeted biopsy may be limited to Asian-American men with PI-RADS 4-5 with 16\%-55\% detection of clinically significant prostate cancer compared with PI-RADS 3-5 in non-Asian-Americans with $15 \%-67 \%$ detection of clinically significant prostate cancer. Furthermore, our results have significant implications for other settings which rely on prostate MRI, such as targeting for prostate partial gland ablation and/or surgical planning. Additional investigation into the independent effect of race on MRI-guided treatment outcomes is needed.

Our findings must be interpreted in the context of our study design. We attempted to employ a broad and inclusive categorization of Asian-American race, however East Asian-Americans comprised the majority of study subjects. We were not able to stratify by immigration history (first generation, and so on) or lifestyle such as exercise and diet. As discussed, our cohort comprised both biopsy-naïve men and those with prior negative biopsy, but this was accounted for in adjusted analysis. Prostate MRI scoring is variable, with one study of nine radiologists reviewing the same images demonstrating significant variation in determination of PI-RADS 3, ranging from $5 \%$ to $26 \% .^{29}$ The study benefited from an experienced uroradiologist (DJM) interpreting MRI images. Finally, as a study in New York City with its diverse population, our results may be validated by others through future multi-institutional studies consistent with IDEAL stage 4 recommendations to include regionally or nationally representative populations.

\section{CONCLUSIONS}

Despite evolution of prostate MRI and targeted biopsy through IDEAL stage 3 Assessment, we demonstrate significant overestimation of PI-RADS risk assessment for prostate cancer in Asian-American men with an elevated PSA. Asian-Americans are $70 \%$ less likely to be diagnosed with clinically significant prostate cancer on MRI-targeted biopsy. Moreover, the incremental benefit of systematic biopsy in all men regardless of race was low, but markedly lower in Asian-Americans. Further, Asian-American men with a PI-RADS 3 region of interest may forego biopsy altogether. This has critical implications for other clinical settings for prostate cancer diagnosis and treatment, which is increasingly dependent on prostate MRI assessment.

Acknowledgements The Frederick J and Theresa Dow Wallace Fund of the New York Community Trust supported this research.

Contributors All authors have made substantial contributions to the conception or design of the work, or the acquisition, analysis or interpretation of data and have approved this manuscript.

Competing interests None declared.

Patient consent for publication Not required.

Provenance and peer review Not commissioned; externally peer reviewed. 
Open access This is an open access article distributed in accordance with the Creative Commons Attribution Non Commercial (CC BY-NC 4.0) license, which permits others to distribute, remix, adapt, build upon this work non-commercially, and license their derivative works on different terms, provided the original work is properly cited, appropriate credit is given, any changes made indicated, and the use is non-commercial. See: http://creativecommons.org/licenses/by-nc/4.0/.

\section{REFERENCES}

1. Barentsz JO, Richenberg J, Clements R, et al. ESUR prostate Mr guidelines 2012. Eur Radiol 2012;22:746-57.

2. Siddiqui MM, Rais-Bahrami S, Turkbey B, et al. Comparison of MR/ ultrasound fusion-guided biopsy with ultrasound-guided biopsy for the diagnosis of prostate cancer. JAMA 2015;313:390-7.

3. Ahmed HU, El-Shater Bosaily A, Brown LC, et al. Diagnostic accuracy of multi-parametric MRI and TRUS biopsy in prostate cancer (PROMIS): a paired validating confirmatory study. Lancet 2017;389:815-22.

4. Kasivisvanathan V, Rannikko AS, Borghi M, et al. MRI-Targeted or standard biopsy for prostate-cancer diagnosis. N Engl J Med 2018:378:1767-77.

5. Rouvière $\mathrm{O}$, Puech $\mathrm{P}$, Renard-Penna R, et al. Use of prostate systematic and targeted biopsy on the basis of multiparametric MRI in biopsy-naive patients (MRI-FIRST): a prospective, multicentre, paired diagnostic study. Lancet Oncol 2019;20:100-9.

6. Hirst A, Philippou Y, Blazeby J, et al. No surgical innovation without evaluation: evolution and further development of the ideal framework and recommendations. Ann Surg 2019;269:211-20.

7. Grossman DC, Curry SJ, Owens DK, et al. Screening for prostate cancer: US preventive services Task force recommendation statement. JAMA 2018;319:1901-13.

8. Colby SL, Ortman JM. Projections of the size and composition of the US population: 2014 to 2060. In: Current population reports, 2014: 25-1143.

9. Siegel RL, Miller KD, Jemal A. Cancer statistics, 2016. CA Cancer J Clin 2016;66:7-30.

10. Nam RK, Saskin R, Lee Y, et al. Increasing hospital admission rates for urological complications after transrectal ultrasound guided prostate biopsy. J Urol 2010;183:963-9.

11. Loeb S, Carter HB, Berndt SI, et al. Complications after prostate biopsy: data from SEER-Medicare. J Urol 2011;186:1830-4.

12. Halpern JA, Sedrakyan A, Dinerman B, et al. Indications, utilization and complications following prostate biopsy: new York state analysis. J Urol 2017;197:1020-5.

13. Patel N, Halpern JA, Kasabwala K, et al. Multiple regions of interest on multiparametric magnetic resonance imaging are not associated with increased detection of clinically significant prostate cancer on fusion biopsy. J Urol 2018;200:559-63.

14. Weinreb JC, Barentsz JO, Choyke PL, et al. PI-RADS Prostate Imaging - Reporting and Data System: 2015, Version 2. Eur Urol 2016;69:16-40.

15. Lauderdale DS, Kestenbaum B. Asian American ethnic identification by surname. Popul Res Policy Rev 2000;19:283-300.

16. Patel N, Cricco-Lizza E, Kasabwala K, et al. The role of systematic and targeted biopsies in light of overlap on magnetic resonance imaging ultrasound fusion biopsy. Eur Urol Oncol 2018;1:263-7.

17. Miller BA, Chu KC, Hankey BF, et al. Cancer incidence and mortality patterns among specific Asian and Pacific Islander populations in the U.S. Cancer Causes Control 2008;19:227-56.

18. Tran HN, Li Y, Udaltsova N, et al. Risk of cancer in Asian Americans: a Kaiser Permanente cohort study. Cancer Causes Control 2016;27:1197-207.

19. Raymundo EM, Rice KR, Chen Y, et al. Prostate cancer in Asian Americans: incidence, management and outcomes in an equal access healthcare system. BJU Int 2011;107:1216-22.

20. Yatani R, Chigusa I, Akazaki K, et al. Geographic pathology of latent prostatic carcinoma. Int J Cancer 1982;29:611-6.

21. Zheng J, Yang B, Huang T, et al. Green tea and black tea consumption and prostate cancer risk: an exploratory meta-analysis of observational studies. Nutr Cancer 2011;63:663-72.

22. Yan L, Spitznagel EL. Soy consumption and prostate cancer risk in men: a revisit of a meta-analysis. Am J Clin Nutr 2009;89:1155-63.

23. Takata R, Akamatsu S, Kubo M, et al. Genome-Wide association study identifies five new susceptibility loci for prostate cancer in the Japanese population. Nat Genet 2010;42:751-4.

24. Bechis SK, Carroll PR, Cooperberg MR. Impact of age at diagnosis on prostate cancer treatment and survival. JCO 2011;29:235-41.

25. Muralidhar V, Ziehr DR, Mahal BA, et al. Association between older age and increasing Gleason score. Clin Genitourin Cancer 2015;13:525-30.

26. Thompson IM, Ankerst DP, Chi C, et al. Assessing prostate cancer risk: results from the prostate cancer prevention trial. J Natl Cancer Inst 2006;98:529-34.

27. Cool DW, Romagnoli C, Izawa JI, et al. Comparison of prostate MRI-3D transrectal ultrasound fusion biopsy for first-time and repeat biopsy patients with previous atypical small acinar proliferation. Can Urol Assoc J 2016;10:342-8.

28. Kim SJ, Vickers AJ, Hu JC. Challenges in adopting level 1 evidence for multiparametric magnetic resonance imaging as a biomarker for prostate cancer screening. JAMA Oncol 2018;4.

29. Sonn GA, Fan RE, Ghanouni P, et al. Prostate magnetic resonance imaging interpretation varies substantially across radiologists. Eur Urol Focus 2017. doi:10.1016/j.euf.2017.11.010. [Epub ahead of print: 6 Dec 2017]. 